\title{
A Contour Fitting Algorithm for Rapid Prototyping Technology
}

\author{
Ge Wu \\ Department of Electronic Engineering, Changchun University of Science and Technology \\ 130022,Changchun, China \\ wuge1900zy@sohu.com
}

Keywords: RP/M; ellipse curve; slicing

\begin{abstract}
This paper presents a fine approach to improve the process planning of Rapid Prototyping/Manufacturing (RP/M) .Corrected curves were introduced to maintain the accuracy of slicing layers. A mixed algorithm combined by tangent line and ellipse fitting method was developed to balance accuracy and build time. Through experiment it has been shown that the algorithm effectively build points sets, and draw the ellipse curves near the original figure.
\end{abstract}

\section{Introduction}

Rapid Prototyping and Manufacturing is a kind of advanced manufacturing technology which was developed for the last 20 years.In recent years, research on the curve shape has made a lot of results in theory and application,but how to make curve fitting of scattered data points, how to construct the new model of shape optimization is still the focus of the parties.

The common methods consist of heuristic search,local edge connectivity,Hough transform,curve fitting and so on..Large scanning spacing results in uncertain layer information,that will affect the rapid prototype manufacturing.To improve the contour information layer and to achieve a reasonable transition layer, it is necessary to interpolate the fault data obtained.

\section{Basic Methods}

\section{The slicing method.}

For the general consideration, the slicing process is always inevitable in rapid prototyping technology, the efficiency of slicing directly affect the entire manufacturing process, whether slicing is accurate or not is related to all aspects. In recent years, research on the curve shape has made a lot of results in theory and application, but how to make curve fitting of scattered data points, how to construct the new model of shape optimization is still the focus of the parties.

In recent years, research on the curve shape has made a lot of results in theory and application, but how to make curve fitting of scattered data points, how to construct the new model of shape optimization is still the focus of the parties. For the general consideration, the slicing process is always inevitable in rapid prototyping technology, the efficiency of slicing directly affect the entire manufacturing process, whether slicing is accurate or not is related to all aspects ${ }^{[1]}$.

The existing direct slicing algorithm of CAD model and STL file format. The hierarchical contour line is curve circle. STL file format has been compatible with most softwares is currently the most widely used standard lattice Type.

Due to the characteristics of the STL file format itself, the slice contour information contains a lot of redundant data, these redundant data affect the future information processing, but also affect the part processing, causing the manufacturing process unstable.

Two dimensional profile reconstruction of ellipse reconstruction.

After collecting and recording the corresponding relationship of all triangles points between contour lines, the reconstruction of the 3D object is finished. The algorithm has the following characteristics: Through coordinate transformation, it solves the non strict coplane of the vertexes in the the same contour and non strict parallel of adjacent contour lines. 
Edges mainly exists in the areas between target, background and objectives, These dramatic changes in gray mutations constitute the image edge,it is easily seen that the phenomenon of edge lack or edge repeat in the same layer contour, which produce profile precision loss.contour tracking is search along the edge of the image,the contour coordinates of points are stored in the column,each contour is represented as a sequence of points ${ }^{[2]}$.

It is easily seen that the phenomenon of edge lack or edge repeat in the samelayer contour, which produce profile precision loss.contour tracking is search along the edge of the image,the contour coordinates of points are stored in the column,each contour is represented as a sequence of points.Due to the presence of noise, the contour will have a gap edge, In RP\&M contour must be closed,so the gaps must be filled.

\section{Proposed algorithm}

\section{The slicing process.}

The basic idea of the algorithm is:The contour lines were grouped according to the height of scanning lines, then through the intersection calculation, crossover points of the intersection line and contour line are gotten.Finally, we connect the points according to a certain order.In the intersection computation,the points are stored according to $\mathrm{x}$ value and $\mathrm{y}$ value that could improve the the execution efficiency, Because of the complexity of the shape, the optimal control algorithm point is not always consistent with the actual situation.if the incision is not conductive to the good reconstruction of 3D objects, you can allows the user to give the incision.

Slicing process:

1.Determinate the direction of slicing.

2.Determinate the height of the first tangent plane $\left(\mathrm{h}_{0}\right.$--the height of the first tangent plane )and $\Delta \mathrm{h}$ layer thickness

3.Connect the lines to form a closed contour.

4.hn=hn-1+ $\mathrm{h}$ ( layer thickness),go to 2 .

\section{Curve fitting.}

At present,curve and surface modeling has formgeometric theory system frame based on approximation, interpolation, fitting.Ellipse fitting is one of the most commonly used method in curve fitting,especially in fitting edge data points.But for the points in small distance, high curvature elliptical shape is too narrow, which will lead to large deviation.

First, canny operator is used for edge detection,are the pixel derivative,the formula of calculating the gradient value of the image in the of $G(x, y)$ is as following:

$$
\mathrm{G}(\mathrm{x}, \mathrm{y})=\left|f_{x}(\mathrm{x}, \mathrm{y})\right|+\left|f_{y}(\mathrm{x}, \mathrm{y})\right|
$$

Then we search for and connect neighboring edge points and then gradually detect all boundary points ${ }^{[3]}$,from the beginning of track initiation, the search direction is along the top left of the initial definition.If the pixel of the upper left is black, then take it as a new boundary point,otherwise the tracking rotates clockwise $45^{\circ}$ until I find a new boundary points,continue in the same way until the return to the starting point.Small curvature part of contour can be lined by tangent method.

The common aspheric curve is described as follow ${ }^{[4]}$ :

$$
x=\frac{c y^{2}}{1+\sqrt{1-(K+1) c^{2} y^{2}}}+d y^{4}+e y^{6}+\ldots
$$

After the detection of closed contour line,corner points detection is executed.In multi ellipse case,if there are no intersection the elliptic arc will be taken by boundary tracking,else maxima of curvature is corner point ${ }^{[5]}$.

This paper uses Floater ${ }^{[6]}$ calculation method of mean center of gravity,Let $\mathrm{P}$ as a star polygon, $\mathrm{q}$ can be expressed as the combination of the P's center of gravity.the expression of the center of gravity is: 


$$
\begin{array}{r}
q=\sum_{i=1}^{n} \lambda_{i} p_{i} \\
\sum_{i=1}^{n} \lambda_{i}=1
\end{array}
$$

The basic idea of the algorithm:

(1)First is to select starting point, end point, and to establish point set,shown as (a)

(2)Connect the starting point and end point to get a line Ps (starting point) and get perpendicular bisector,shown as(b).

(3)Keep the points set close to the bisector,shown as (c).

(4)Calculate the the weighted average of kept points set,then format a point P,shown as (d).

$$
P=\sum_{0}^{n} P_{\text {edge }}(i, j)
$$

(5)Calculate the height of the chord, then calculate the radius,draw the circle by the radius,shown as (e)(f).

$$
r=\frac{a^{2}+h^{2}}{2 h}
$$

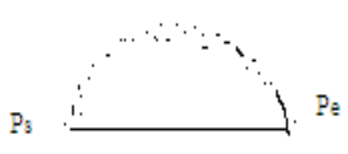

(a)scatterd points

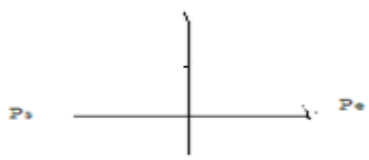

(d)format a point

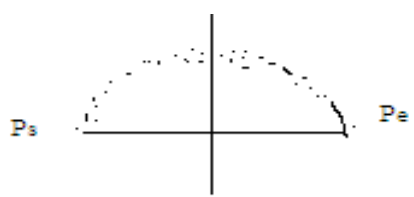

(b)Perpendicular bisector

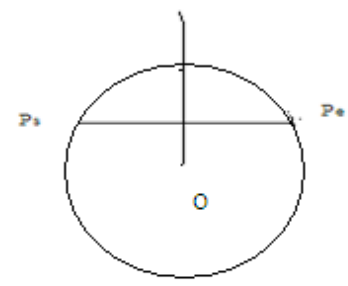

(e) draw the circle

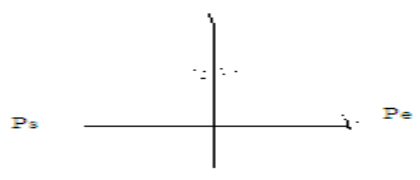

(c)points near bisector

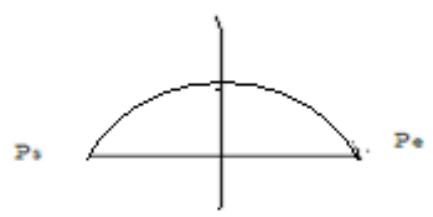

0

(f) keep the arc

Fig 1. Fitting process

\section{Experimental Results}

We use a part with arc and straight lines as examples in figure 2,In figure (b),we only use ellipse fitting,the result shows it fit well in arc part,but it ignores fold line part so that the details can not be described.In figure(d),we combine the tangent line method with ellipse method,contour can be well described. 


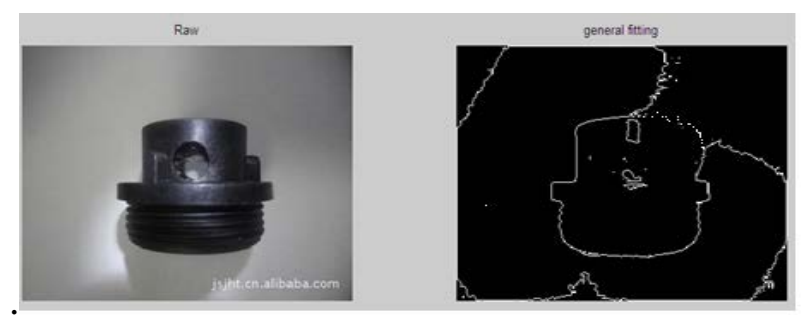

(a)before fitting

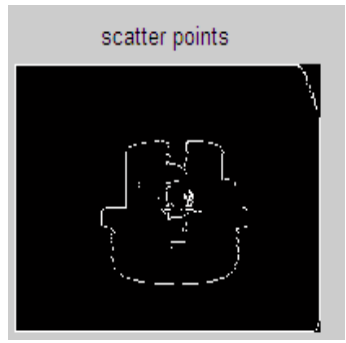

(c)scatter points

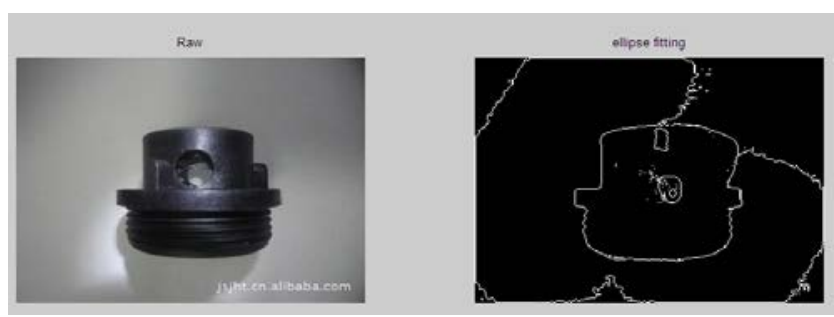

(b)ellipse fitting

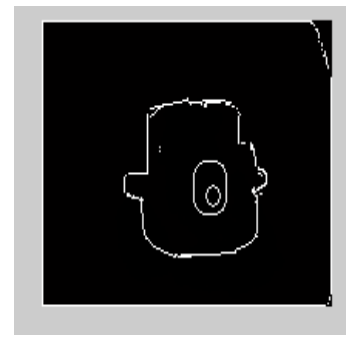

(d) tangent line

Figure 2.comparation of before fitting and ellipse fitting

\section{Conclusion}

This algorithm is suitable for fitting various arc curve, the time complexity of the algorithm will be greatly reduced. But for a straight line and a fold line it is not well applicable,and for big curve it need more experiments.so before fitting we should have a pre-sentence for improvement.

\section{References}

[1] Ming Chih Huang, Ching-Chih Tai. The pre-processing of data point for curve fitting in reverse engineering[J]. International Journal of Advanced Manufacturing Technology,2000(16): 635642.

[2] CANNY J.F.A computational approach to edge detection.IEEE Trans.On Patten Analysis and Machine Intelllgence,1986,8(6):679 - 698.

[3] KAWAGUCHI T,NAGATAR R.Ellipse detection using grouping of edges into line-support regions.International Conference on lmage Processing,1998,3:70 - 74.

[4] Bodo Rosenhahn, Christian Perwass, Gerald Sommer. Pose Estimation of 3D Free-form Conformal Geometry [J].2001.

[5]Ho-Chan Kim, Seok-Hee Lee. Reduction of post-processing for stereolithography systems by fabrication-direction optimization[J].Computer-Aided Design, 2005,37:711-725. 